\title{
PERFIL LIPIDICO DE DIETAS E SUA RELAÇÃO COM OS NÍVEIS DE COLESTEROLEMIA EM ESCOLARES DE UMA ESCOLA PÚBUCA DE CAMPINAS, SÃO PAULO, (BRASIL)1
}

\author{
UPID PROFILE OF DIETS AND THE CORREIATION WTH SERUM CHOLESTEROL IN \\ SCHOOLCHIDREN FROM PUBUC SCHOOL IN CAMPINAS, SÃO PAULO (BRAZL)
}

Ely Catarina de MOURA ${ }^{2}$

Jaqueline Gimos SONAT ${ }^{3}$

\begin{abstract}
RESUMO
Este estudo foi conduzido com 194 escolares com o objetivo de identificar o perfil lipídico de suas dietas, relacionando-os com o colesterol plasmático. Foi realizado um inquérito alimentar de 24 horas e a dosagem de colesterol e HDL-colesterol. Associação entre lipídios da dieta e do sangue foi analisada por correlação bivariada e regressão logística. A dieta consumida pelos escolares mostrou diferença significativa (teste t), entre os sexos, para a quantidade de carboidratos e de fibra alimentar. Os carboidratos contribuíram com 52,7\% das calorias totais, as proteínas com 14,0\%, os triglicérides com $33,3 \%$, sendo que os ácidos graxos monoinsaturados com $12,0 \%$, os poliinsaturados com $8,5 \%$ e os saturados com 12,5\%. A quantidade de colesterol na dieta foi $176 \mathrm{mg}$ e de fibra alimentar $15 \mathrm{~g}$. $\mathrm{O}$ valor do colesterol plasmático foi $150 \mathrm{mg} /$ dl e do HDL-colesterol $36 \mathrm{mg} / \mathrm{dl}$. Observou-se associação direta entre colesterolemia e percentual de calorias proveniente de triglicerídeos, de ácidos graxos monoinsaturados e poliinsaturados. Encontrou-se associação positiva entre colesterolemia e porcentagem de calorias proveniente de ácidos graxos monoinsaturados. Concluiu-se que o método utilizado neste estudo não foi apropriado ao objetivo proposto devido à baixa prevalência de hipercolesterolemia (4,8\%) entre os escolares estudados. Fatores de respostas individuais devem ser considerados no estudo de associação entre colesterol dietético e plasmático.
\end{abstract}

Termos de indexação: colesterol, lipídios na dieta, nutrição da criança, saúde infantil, estudantes.

\begin{abstract}
This study was carried out with 194 schoolchildren with the purpose of investigating the lipid profile of their diets and the correlation with serum cholesterol. A 24-hour food inquiry was performed and serum cholesterol levels, including HDL-cholesterol, were measured by enzymatic methods. Bivariate correlation and logistic regression were done to find any association between dietary fat consumption and serum cholesterol. The intake of

\footnotetext{
(1) Auxílio Parcial do Conselho Nacional de Desenvolvimento Científico e Tecnológico/CNPq na forma de bolsas de apoio técnico, iniciação científica, aperfeiçoamento e pesquisa. Processos números 300644/92-2, 804563/90-0 e 823035/90-5.

(2) Professora Titular do Departamento de Nutrição da Faculdade de Ciências Médicas da PUC - Campinas, E-mail: lymoura@aleph.com.br

(3) Bolsista de Aperfeiçoamento do Conselho Nacional de Desenvolvimento Científico e Tecnológico/CNPq.
} 
carbohydrates and fiber for boys was bigger than for girls ( $t$ test). The mean of calories supplied by carbohydrates was $52.7 \%$, by proteins was $14.0 \%$, by triglycerides was $33.3 \%$, by monounsaturated fatty acids was $12.0 \%$, by polyunsaturated was $8.5 \%$ and by saturated was $12.5 \%$. The average of cholesterol intake was $176 \mathrm{mg}$ and of fiber was $15 \mathrm{~g}$. The mean level of serum cholesterol was $150 \mathrm{mg} /$ $\mathrm{dl}$ and of HDL-cholesterol was $36 \mathrm{mg} / \mathrm{dl}$. The serum cholesterol was related to calories from triglycerides, monounsaturated and polyunsaturated fatty acids. Logistic regression showed association between monounsaturated fatty acids and high cholesterol level. In conclusion, the method used in this study was not appropriate to identify relationship between dietary fat and serum cholesterol because the prevalence of hypercholesterolemia in the study population (4.8\%) was low. Individual answer factors showed be considered in the study of association between dietary fat and serum cholesterol.

Index Terms: cholesterol, dietary fats, child nutrition, child health, studentes.

\section{INTRODUÇÃO}

O risco de hipercolesterolemia tem sido associado a vários fatores, como idade, sexo, pressão arterial, índice ponderal ou índice de massa corporal, tabagismo, história familiar de doença cardiovascular, uso de contraceptivo oral e dieta (BADRUDDIN et al., 1994; BENUCK et al., 1995; MYERS et al., 1995).

A dieta, ainda que haja alguma controvérsia (PURATH et al., 1995), tem assumido um papel primordial na medida em que o alto teor de colesterol, o baixo consumo de fibra alimentar, a alta proporção de calorias lipídicas e ácidos graxos saturados têm sido associados com o aumento da colesterolemia (VOBECKY et al., 1995; WAJID ALI et al., 1995). Por outro lado, a redução da colesterolemia, mesmo que pequena, parece ser eficiente na diminuição dos índices de mortalidade por doenças cardiovasculares (McCABE, 1993).

Na medida em que, no Brasil as mortes por doenças cardiovasculares contribuem significativamente como grupo de causa de morte em todas as regiões (DUNCAN et al., 1992; SICHIERI et al., 1992), é necessário avaliar o perfil lipídico das dietas e a sua relação com os níveis de colesterol do sangue, uma vez que o colesterol dietético contribui aproximadamente com $15 \%$ na formação do colesterol endógeno (FREDRICKSON et al., 1973) e que, principalmente, a intervenção educativa precoce pode contribuir para um melhor estado de saúde. Considerando, ainda, que o nível de colesterolemia na infância é um fator preditivo do nível de colesterolemia na vida adulta (KEMPER et al., 1990; WEBBER et al., 1991), este estudo teve o propósito de analisar o perfil lipídico de dietas consumidas por escolares e sua relação com a concentração de colesterol no sangue.

\section{MATERIAL E MÉTODOS}

Este trabalho é parte do projeto "Saúde escolar: diagnóstico, monitorização e prevenção" desenvolvido por uma equipe multidepartamental, junto ao Centro de Saúde Escola Jardim Campos Elíseos da Pontifícia Universidade Católica de Campinas (PUC-Campinas), e aprovado pelo Comitê de Ética da Faculdade de Ciências Médicas da PUC-Campinas (MOURA et al., 1997).

Campinas localiza-se cerca de 100 quilômetros ao noroeste da cidade de São Paulo e caracteriza-se por apresentar uma população bastante heterogênea, variando dos mais altos aos mais baixos estratos sociais. É constituída por aproximadamente 200 mil domicílios, contando com uma população estimada em 800 mil habitantes, conforme Censo de 1991. O município conta com rede de água, luz, esgoto e asfalto. Entretanto, apresenta alguns bolsões de pobreza (espaço aglomerado, caracterizado por favelas em processo de urbanização) com precárias condições de saneamento básico e baixa renda familiar (MOURA et al., 1997).

A população do estudo foi composta pelas crianças matriculadas na primeira série da Escola Estadual de Primeiro Grau André Fort; nos anos de 1994 e 1995. Foram identificadas 231 crianças, sendo que 194 consentiram em participar do estudo. Em 1994, as crianças foram divididas em quatro 
classes, num total de 110 alunos; em 1995 em 3 classes, num total de 84 alunos. A participação no estudo foi voluntária e o sigilo dos dados garantido pela equipe de pesquisadores, sendo o trabalho iniciado após o consentimento da escola e, por escrito, dos responsáveis pela criança. Os escolares, acompanhados por seus responsáveis foram convidados para avaliação clínica, nutricional, postural e das condições socioeconômicas. Eles foram orientados sobre a necessidade dos exames bioquímicos e coleta de materiais (sangue, urina e fezes).

O inquérito alimentar recordatório de 24 horas foi aplicado em entrevista com o escolar, completada, posteriormente, com a participação dos responsáveis pela alimentação da criança. O cálculo diário da ingestão dietética foi realizado pelo programa AVANU 4 , com base nas tabelas de composição de alimentos do Estudo Nacional da Despesa Familiar (INSTITUTO..., 1977) para energia e macronutrientes e McCance \& Widdowson's (HOLLAND et al., 1992) para os ácidos graxos, colesterol e fibra. Foram calculadas as quantidades de energia, proteínas, carboidratos, ácidos graxos saturados, monoinsaturados e poliinsaturados, colesterol e fibra alimentar.

O sangue foi colhido por punção venosa, de cada escolar, após jejum mínimo de 12 horas. As amostras de sangue foram processadas no Laboratório de Análises Clínicas e Toxicológicas do Curso de Ciências Farmacêuticas da PUC-Campinas. O colesterol e HDL-colesterol foram determinados pelo método colorimétrico-enzimático (NATIONAL..., 1974).

Foram calculadas a média (M) e o desvio padrão (DP) das variáveis em estudo, conforme o sexo. Para a análise estatística foi utilizado o teste “t”, na comparação de médias. Foi realizada a correlação bivariada utilizando-se o coeficiente de Pearson para verificar a associação entre fatores lipídicos da dieta: percentual de calorias proveniente de lipídeos, de ácidos graxos monoinsaturados, poliinsaturados e saturados, além da fibra alimentar e do colesterol dietético e sanguíneo (colesterol total e HDL-colesterol). Para determinar se a associação entre estes fatores persistia, após ajuste para as variáveis, regressão logística foi executada, considerando-se o percentil 75 como limite entre alta e baixa colesterolemia (MANLY, 1986).

Os dados foram processados utilizando-se o programa Statistical Package for Social Sciences (STATISTICAL..., 1992), considerando intervalo de confiança de $95 \%$ e nível de significância de 5\% $(\mathrm{p}<0,05)$.

Tabela 1. Características das dietas conforme o sexo dos escolares, 1994/95.

\begin{tabular}{|c|c|c|c|c|c|}
\hline \multirow[t]{2}{*}{$\mathrm{M} \pm \mathrm{DP}$} & \multicolumn{2}{|c|}{ Sexo } & \multirow[t]{2}{*}{ Total } & \multicolumn{2}{|c|}{ Valor de } \\
\hline & Feminino & Masculino & & $\mathrm{t}$ & $\mathrm{p}$ \\
\hline Energia (kcal) & $1943 \pm 646$ & $2010 \pm 678$ & $2033 \pm 666$ & 1,85 & 0,07 \\
\hline Proteínas (g) & $69 \pm 35$ & $74 \pm 30$ & $71 \pm 33$ & 1,24 & 0,25 \\
\hline Carboidratos (g) & $254 \pm 102$ & $284 \pm 98$ & $269 \pm 101$ & 2,08 & 0,04 \\
\hline Triglicerídes (g) & $73 \pm 28$ & $76 \pm 34$ & $75 \pm 31$ & 0,70 & 0,49 \\
\hline Ácidos graxos monoinsaturados (g) & $26 \pm 11$ & $28 \pm 14$ & $27 \pm 12$ & 0,99 & 0,32 \\
\hline Ácidos graxos poliinsaturados (g) & $19 \pm 10$ & $19 \pm 12$ & $19 \pm 11$ & 0,08 & 0,94 \\
\hline Ácidos graxos saturados (g) & $27 \pm 11$ & $29 \pm 12$ & $28 \pm 12$ & 1,02 & 0.31 \\
\hline Colesterol (mg/dl) & $181 \pm 186$ & $174 \pm 126$ & $176 \pm 157$ & 0,31 & 0,76 \\
\hline Fibra alimentar (g) & $13 \pm 9$ & $16 \pm 8$ & $15 \pm 9$ & 2,67 & 0,008 \\
\hline
\end{tabular}

\section{RESULTADOS}

Estes resultados são decorrentes de um estudo transversal, descritivo e analítico, que vem sendo desenvolvido, anualmente, junto aos escolares da primeira série da Escola Estadual de Primeiro Grau André Forti (EEPGAF) na área de cobertura do Centro de Saúde Escola, atendendo à sua proposta de atenção global à saúde do escolar. A idade média

(2) Desenvolvido por Manfredo Rassi Tabacniks e Erly Catarina de Moura. 
dos escolares estudados foi 7,8 $\pm 0,5$ anos, a renda familiar mensal per capita foi 1,6 $\pm 1,1$ salários mínimos (excluindo-se um caso com renda 7,1, um com 13,3 e dois com 14,3 salários mínimos, cujos provedores eram empresários), 47,9\% eram do sexo feminino e $52,1 \%$ do sexo masculino.

A Tabela 1 mostra as características das dietas consumidas pelos escolares. Apenas a quantidade de carboidratos e de fibra alimentar foram estatisticamente diferente entre os sexos. A média do valor energético encontrada para estas crianças foi de $2033 \pm 666 \mathrm{kcal}$. Desse valor calórico total, as proteínas contribuíram com $14,0 \%$, os carboidratos com $52,7 \%$ e os triglicerídeos com $33,3 \%$, estes últimos divididos em ácidos graxos monoinsaturados (12,0\%), poliinsaturados (8,5\%) e saturados $(12,5 \%)$.

Quanto à concentração de colesterol no sangue, encontrou-se $156 \pm 27 \mathrm{mg} / \mathrm{dl}$ para as meninas e $145 \pm 27 \mathrm{mg} / \mathrm{dl}$ para os meninos; para o HDL-colesterol os valores foram $36 \pm 7$ e $36 \pm 8 \mathrm{mg} / \mathrm{dl}$, respectivamente para o sexo feminino e masculino. Não foi observada nenhuma diferença significativa entre os sexos.

A correlação mostrou que a colesterolemia se associou positivamente com percentual de calorias proveniente de triglicerídeos $(\mathrm{p}=0,006)$, de ácidos graxos monoinsaturados $(\mathrm{p}=0,005)$ e poliinsaturados $(p=0,04)$; e negativamente com quantidade de colesterol ingerido na dieta $(p=0,02)$. HDL-colesterol se associou positivamente com porcentagem de calorias proveniente de lipídios $(\mathrm{p}=0,003)$, de ácidos graxos monoinsaturados $(\mathrm{p}=0,009)$, poliinsaturados $(\mathrm{p}=0,03)$ e saturados $(\mathrm{p}=0,024)$.

Adotando-se o percentil 75 da distribuição de colesterol do sangue (168 mg/dl), encontrou-se diferença para porcentagem de calorias proveniente de ácidos graxos monoinsaturados, cuja média para o baixo colesterol foi $5,1 \pm 1,6 \%$ e para o alto $6,0 \pm$ $2 \%(\mathrm{t}=2,77, \mathrm{p}=007)$. A regressão logística, ajustada para os componentes lipídicos da dieta, apontou associação direta entre colesterolemia e porcentagem de calorias proveniente de ácidos graxos monoinsaturados $(\mathrm{p}<0,004)$.

\section{DISC USSÃO E CONCLUSÃO}

Dados de várias pesquisas têm relatado alta colesterolemia em crianças e adolescentes, levando muitos estudiosos a considerar a necessidade de prevenção pediátrica das doenças cardiovasculares (PORKKA et al., 1991; VIIKARI et al., 1991; POLONSKY et al., 1993; NEWMAN et al., 1995).

Neste estudo, a concentração de colesterol no sangue para as meninas (156 $\pm 27 \mathrm{mg} / \mathrm{dl})$ e meninos $(145 \pm 27 \mathrm{mg} / \mathrm{dl})$ foi menor do que a referida em outros trabalhos. Valores encontrados na faixa etária semelhante à estudada foram respectivamente para as meninas e os meninos, $185 \pm 35$ e $178 \pm 31 \mathrm{mg} / \mathrm{dl}$ (BADRUDDIN et al., 1991), $160 \pm 28$ e $155 \pm$ $27 \mathrm{mg} / \mathrm{dl}$ (WONG et al., 1992), 173mg/dl e $168 \mathrm{mg} / \mathrm{dl}$ (WEBBER et al., 1995).

O HDL-colesterol, considerado fator de proteção contra as doenças cardíacas, ficou abaixo dos valores citados em outros estudos: $36 \pm 7 \mathrm{mg} / \mathrm{dl}$ para o sexo feminino e $36 \pm 8 \mathrm{mg} / \mathrm{dl}$ para o sexo masculino. RIFKIND \& SEGAL (1983) referem $55 \mathrm{mg} / \mathrm{dl}$ para ambos os sexos e WEBBER et al. (1995) 50,2mg/dl para as meninas e 52,7mg/dl para os meninos.

Quanto as características da dieta, os dados mostraram que o consumo médio de fibras foi considerado adequado, segundo recomendação de WILLIAMS (1995), que indica 12 a 13 gramas para a idade média deste estudo). A ingestão média de lipídios está acima da recomendação nacional (20 a 25\%), e as proporções dos ácidos graxos, no total de triglicerídeos, não foram adequadas (36\% de monoinsaturados: variação de 18 a 53\%; 25\% de poliinsaturados: variação de 5 a $47 \%$, e $38 \%$ de saturados: variação de 20 a 59\%). A quantidade média ingerida de colesterol manteve-se nos padrões internacionais recomendados. Todavia, 9,9\% dos escolares consumiam mais que $250 \mathrm{mg}$ de colesterol (limite recomendado por BROWN \& BRENTON, 1994) e $28,0 \%$ mais que $300 \mathrm{mg}$ (valor recomendado pelo Instituto Nacional de Saúde dos Estados Unidos citado por BROWN \& BRENTON, 1994).

Das variáveis lipídicas da dieta associadas com colesterolemia, apenas a relação com o percentual de calorias proveniente de triglicerídeos 
foi coerente com o esperado. A quantidade de colesterol e o percentual de calorias proveniente de ácidos graxos monoinsaturados e poliinsaturados apresentaram resultado oposto ao esperado. A associação direta entre colesterolemia e porcentagem de calorias proveniente de ácidos graxos monoinsaturados indica que o método utilizado neste estudo não foi adequado à proposta inicial. A amostra foi pequena para os poucos casos de hipercolesterolemia entre os escolares desta região. Sugere-se, para um outro estudo ampliar a amostra ou utilizar o desenho epidemiológico caso-controle.

A controvérsia entre a associação do colesterol dietético e do plasmático, citada na literatura, pode ser explicada pela interação entre o colesterol da dieta e outros nutrientes, por respostas diferenciadas quanto a síntese e excreção do colesterol endógeno e por um determinado limite de colesterolemia, a partir do qual haveria influência do colesterol dietético (KESTIN et al., 1989).

Entretanto, encontramos 22,8\% das crianças com colesterol plasmático acima de $170 \mathrm{mg} / \mathrm{dl}$, valor recomendado pelo National Institute of Health (NATIONAL..., 1985) para o aconselhamento dietético. O National Institutes of Health aconselha, ainda, a supervisão rotineira dos indivíduos com níveis de colesterol plasmático acima de $185 \mathrm{mg} / \mathrm{dl}$ e medicação, quando não houver resposta às mudanças dietéticas e aumento da atividade física para aqueles com colesterol acima de $200 \mathrm{mg} / \mathrm{dl}$. Neste estudo, os níveis de colesterol no plasma atingiram valores superiores a $185 \mathrm{mg} / \mathrm{dl}$ e $200 \mathrm{mg} / \mathrm{dl}$ em $16,8 \%$ e $4,8 \%$ dos escolares, respectivamente.

Em conseqüência deste trabalho, todas as crianças com colesterol acima de $170 \mathrm{mg} / \mathrm{dl}$ foram consultadas pelo Serviço de Nutrição do Centro de Saúde Escola, mantendo-se retornos mensais. Pretende-se, ainda, desenvolver um trabalho de educação em saúde, com enfâse na nutrição junto à comunidade local em colaboração com a Escola estudada. Afinal, os problemas que uma dieta inadequada pode trazer para a saúde das crianças são muitos, a maioria a longo prazo, mas evitáveis se a dieta for corrigida em tempo.

\section{REFERÊNCIAS BIBUOGRÁFICAS}

BADRUDDIN, S.H. KHURSHID, M., MOLLA, A., MANSER, W.W.T., LALANI, R., VELLANI, C.W. Factors associated with elevated serum cholesterol levels in well-to-do Pakistani schoolchildren. Journal of Tropical Medicine and Hygiene, Oxford, v.94, n.2, p.123-129, 1991.

BADRUDDIN, S.H., MOLLA, A., KHURSHID, M., VAZ, S., HASSANALI, S. Cardiovascular risk factors in school children from low middle income families in Karachi. Journal of the Pakistan Medical Association, Karachi, v.44, n.5, p.106-112, 1994.

BENUCK, I., GIDDING, S.S., DONOVAN, M. Year-to-year variability of cholesterol levels in a pediatric practice. Archives of Pediatric and Adolescent Medicine, Chicago, v.149, n.3, p.292-296, 1995.

BROWN, A.C., BRENTON, B. Dietary survey of Hopi Native American elementary students. Journal of the American Dietetic Association, Chicago, v.94, n.5, p.517-522,1994.

DUNCAN, B.B., SCHMIDT, M.I., POLANCZYK, C.A., MENGUE, S.S. Altos coeficientes de mortalidade em populações adultas brasileiras: uma comparação internacional. Revista da Associação Médica Brasileira, São Paulo, v.38, n.3,p.138-144,1992.

FREDRICKSON, D.S., BONNEL, M., LEVY, R.I., ERNST, N. Dietary management of hyperlipo-proteinemia: a handbook for physicians and dietitians. Bethesda : National Institute of Health, 1973. (Publication NIH 73-110).

HOLLAND, B., WELCH, A.A., UNWIN, I.D., BUSS, D.H., PAUL, A.A., SOUTHGATE, D.A.T. McCance \& Widdowson's the composition of foods. 5.ed. Cambridge : The Royal Society of Chemistry, 1992. $462 \mathrm{p}$. 
INSTITUTO BRASILEIRO DE GEOGRAFIA E ESTATÍSTICA. Estudo nacional de despesa familiar: tabelas de composição de alimentos. Rio de Janeiro, 1977. 202p. (Publicações especiais, v.3, t.1).

KEMPER, H.C.G., SNEL, J., VERSCHUUR, R., ESSEN, L.S. Tracking of health and risk indicators of cardiovascular diseases from teenager to adult: Amsterdam growth and health study. Preventive Medicine, New York, v.19, n.6, p.642-655, 1990.

KESTIN, M., CLIFTON, P.M., ROUSE, I.L., NESTEL, P.J. Effect of dietary cholesterol in normolipidemic subjects is not modified by nature and amount of dietary fat. American Journal of Clinical Nutrition, Bethesda, v.50, n.3, p.528-532, 1989.

MANLY, B.F.J. Multivariate statistical methods. London : Chapman and Hall, 1986. 159p.

McCABE, E.M. Monitoring the fat and cholesterol intake of children and adolescents. J Pediatr Health Care, v.7, n.2, p.61-70, 1993.

MOURA, E.C., NAKAMURA, S.Y., ROMERO, V.S.V. Níveis de colesterol em escolares da primeira série de uma escola pública de Campinas, São Paulo, Brasil. Revista de Nutrição da PUCCAMP, v.10, n.1, p.63-69, 1997.

MYERS, L., COUGHLIN, S.S., WEBBER, L.S., SRINIVASAN, S.R., BERENSON, G.S. Prediction of adult cardiovascular multifactorial risk status from childhood risk factor levels. The Bogalusa Heart Study. American Journal of Epidemiology, Baltimore, v.142, n.9, p.918924, 1995.

NATIONAL INSTITUTE OF HEALTH. Manual of laboratory operations. Washington DC, 1974. v.1 (DHEW Publication, 75-628).
NATIONAL INSTITUTE OF HEALTH. High blood cholesterol in adults. Washington DC, 1985. (NCEP Publication, 88-2925).

NEWMAN, T.B., GARBER, A.M., HOLTZMANA, N.A., HULLEY, S.B. Problems with the report of the Expert Panel on blood cholesterol levels in children and adolescents. Archives of Pediatric and Adolescent Medicine, Chicago, v.149, n.3, p.241-247, 1995.

POLONSKY, S.M., BELLET, P.S., SPRECHER, Primary hyperlipidemia in a pediatric population: classification and effect of dietary treatment. Pediatrics, Evanston, v.91, n.1, p.9296, 1993.

PORKKA, K.V.K., VIIKARI, J.S.A., AKERBLOM, H.K. Tracking of serum HDL-cholesterol and other lipids in children and adolescents: the cardiovascular risk in young Finns study. Preventine Medicine, New York , v.20, n.6, p.713-724, 1991.

PURATH, J., LANSINGER, T., RAGHEB, C. Cardiac risk evaluation for elementary school children. Public Health Nursing, Boston, v.12, n.3, 189-195, 1995.

RIFKIND, B.M., SEGAL, P. Lipid Research Clinics Program reference values for hyperlipidemia and hypolipidemia. JAMA, Chicago, v.250, n.14, p.1869-1872, 1983.

SICHIERI, R., LOLIO, C.A., CORREIA, V.R., EVERHART, J.E. Variações geográficas no padrão de mortalidade proporcional por doenças crônico-degenerativas no Brasil. Revista de Saúde Pública, São Paulo, v.26, n.6, p.424430, 1992.

STATISCAL PACKAGE FOR THE SOCIAL SCIENCES FOR WINDOWS. Advanced statistics. Release 5. Chicago, 1992. 580 p. 
VIIKARI, J., RÕNNEMAA, T., SEPPÃNEN, A., MARNIEMI, J., PORKKA, K., RÃSÃNEN, L., UHARI, M., SALO, M.K., KAPRIO, E.A., NUUTINEN, E.M., PESONEN, E., PIETIKÃINEN, M., DAHL, M., AKERBLOM, H.K. Serum lipids and lipoproteins in children adolescents and young adults in 1980-1986. Annals of Medicine, Helsink, v.23, n.1, p.5359,1991.

VOBECKY, J.S., NORMAND, L. Risk and benefits of low fat intake in childhood. Annals of Nutrition and Metabolism, Basel, v.39, n.2, p.124-133, 1995.

WAJID ALI, S., BUCH, N.A., MASOOD, H. Serum lipid profile in Kashmiri children. Indian Journal of Physiology and Pharmacology, New Delhi, v.39, n.1, p.55-58, 1995.

WEBBER, L.S., ORGANIAN, V., LUEPKER, R. V., FELDMAN, H.A., STONE, E.J., ELDER, J.P., PERRY, C.L., NADER, P.R., PARCEL, G.S., BROYLES, S.L., McKINLAY, S.M.
Cardiovascular risk factors among third grade children in four regions of the United States. American Journal of Epidemiology, Baltimore, v.141, n.5, p.428-439, 1995.

WEBBER, L.S., SRINIVASAN, S.R., WATTIGNEY, W.A., BERENSON, G.S. Tracking of serum lipids and lipoproteins from childhood to adulthood. American Journal of Epidemiology, Baltimore, v.133, n.9, p.884899, 1991.

WILLIAMS, C.L. Importance of dietary fiber in childhood. Journal of the American Dietetic Association, Chicago, v.95, n.10, p.1140-1146, 1995.

WONG, N.D., HEI, T.K., QAQUNDAH, P.Y., DAVIDSON, D.M., BASSIN, S.L., GOLS, K.U. Television viewing and pediatric hypercholesterolimia. Pediatrics, Evanston, v.90, n.1, p.7579, 1991.

Recebido para publicação em 13 de fevereiro e aceito em 19 de setembro de 1997. 